\title{
Path Loss Models in Viaduct and Plain Scenarios of the High-speed Railway
}

\author{
Hong Wei, Zhangdui Zhong, Ke Guan, Bo Ai \\ State Key Laboratory of Rail Traffic Control and Safety \\ Beijing Jiaotong University, 100044, Beijing, China \\ E-mail: xingzhihappy@163.com
}

\begin{abstract}
This paper focuses on propagation path loss modeling in viaduct and plain scenarios of the High-speed Railway. The data used for modeling comes from measurement on ZhengzhouXi'an passenger dedicated line with the maximum moving speed of $340 \mathrm{Km} / \mathrm{h}$. Based on the measurement data, tuned Free-space path loss models in these two scenarios are proposed. The performance of the tuned models is compared with that of the Hata model. The evaluation of the models is in terms of mean error, root mean square error and standard deviation of the residuals between the models and measurement. The simulation results and related analysis show better performance of the proposed tuned models compared with the conventional Hata model.
\end{abstract}

Index Terms - path loss; modeling; High-speed Railway; viaduct; plain

\section{INTRODUCTION}

With the rapid development of the High-speed Railway, more and more attention has been paid to the characteristics of the wireless channel in railway scenarios. Among all the characteristics of the High-speed Railway, path loss is a basic one which is of great importance in the design of the Highspeed Railway Communication System.

As we all know, there are lots of models based on the statistical and theoretical analyzes. But none of them are universally applicable. The Hata model is widely used in macrocell scenarios. And also, a lot of work is done to predict the propagation path loss between the transmitter and the receiver in such scenarios as urban or suburban [1], [2]. Unfortunately, some typical scenarios of the High-speed Railway, such as viaduct, plain and cuttings, are few considered.

The propagation path loss modeling methods are in varied forms. Although ray tracing[3] is a more precious method, it's time and memory consuming in the modeling of largescale propagation. Similarly, reference[4] suggests improving the performance of the model by using detailed morphological and building data. But the detailed information needed is hard to obtain. Therefore, statistical models are frequently used. Reference[5] suggests adding the average residual to the present model. However, the path loss exponent remains the same in this way. In practice, obstructions between the transmitter and receiver as well as multipath propagation

This work is supported by the Joint State Key Program of the National Natural Science Foundation of China (No. 60830001), the Program for Changiiang Scholars and Innovative Research Team in University (No. IRT0949) and the State Key Laboratory of Rail Traffic Control and Safety (No. RCS2008ZZ006), Beijing Jiaotong University. will have effects on the value of the path loss exponent[6]. Therefore, the path loss exponents are so important that should be tuned in different scenarios. Most of the references such as[7]-[10] present the path loss exponent and the shadowing to show the large-scale propagation characteristics of some typical scenarios based on measurement. Though precious, these models lose the universality.

To meet the needs of the path loss modeling in the scenarios of the High-speed Railway, model tuning is done based on the measurement data of the Zhengzhou-Xi'an(Zhengxi) Highspeed Railway. In this paper, firstly, measurement environment and the system are introduced. Then, based on the measurement data, tuned Free-Space path loss models are presented in viaduct and plain scenarios, respectively. Finally, performance evaluation is made to check whether these two models can predict the path loss in these two scenarios well.

\section{MEAsurement EnVironment And The System}

\section{A. Measurement Environment}

The measurement has been carried out in Zhengxi Highspeed Railway with a total length of $458 \mathrm{Km}$. And the speed of train is up to $340 \mathrm{Km} / \mathrm{h}$. Usually, there are some typical scenarios along the high speed railway, such as viaduct, cuttings, plain and mountain area. Along Zhengxi railway line, viaduct, which is built highly above the ground, occupies more than $50 \%$ of all the scenarios. The viaduct scenario is showed in Fig.1. As the figure shows, the surface of the viaduct is almost flat. And it is higher than most of the surroundings.

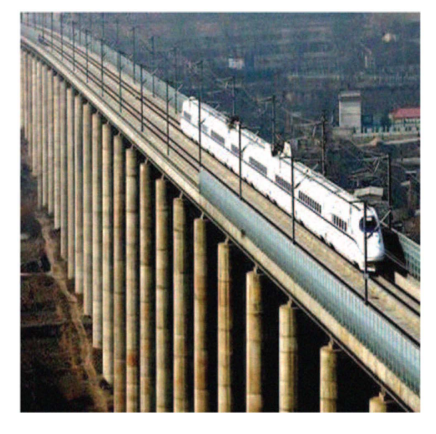

Fig. 1. Viaduct scenario

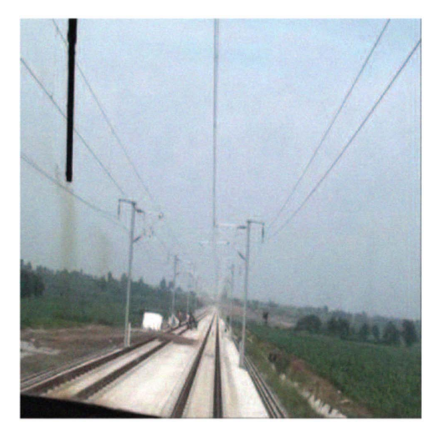

Fig. 2. Plain scenario
In plain scenario, the fluctuate of the ground is small, just like that of the surface of the viaduct. Therefore, the propagation characteristics have something like in these two 
scenarios. Fig.2 shows a typical plain scenario in Zhengxi High-speed Railway.

\section{B. Test System}

The measurement has been taken in the present network on Zhengxi passenger dedicated line. Therefore, the testing frequency is the same as the downlink frequency, which ranges from $930.2 \mathrm{MHz}$ to $933.4 \mathrm{MHz}$ with a bandwidth of $200 \mathrm{KHz}$. The transmitting antennas on the line are mainly cross-polarized with $17 \mathrm{dBi}$ Gain, $65^{\circ}$ horizontal and $6.8^{\circ}$ vertical beam width. The transmitting power of the antennas is $43 \mathrm{dBm}$. And the receiving antenna which is omni-directional is placed on the top of the train, with a height of $3.5 \mathrm{~m}$.

The receiving system, which is shown in Fig.3, is composed of the receiver Griffin, the software and the GPS. The receiving power collected by Griffin and the position information collected by the GPS are given to the software to do some analysis. The sampling rate of Griffin is one sample per $52 \mathrm{~cm}$. And the receiving data between two base stations is called a group in this paper.

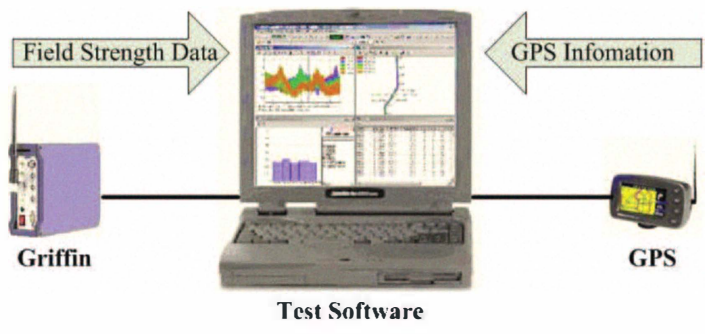

Fig. 3. Test system

\section{Modeling of The PATH Loss in Viaduct AND Plain SCEnARIOS}

\section{A. Measurement Results and Analysis}

Fig. 4 and Fig. 5 show the receiving power with log-distance in viaduct and plain scenarios. And the unit of the distance is $\mathrm{Km}$. The measurement distances are $3 \mathrm{Km}$ and $1.793 \mathrm{Km}$ in these two pictures, respectively. The fast fading is smoothed out according to Lee's law[11].

In the large-scale propagation modeling, the near field data should be deleted. The two figures given here and other measurement results show that the receiving power suffers large fluctuation within $300 \mathrm{~m}$. So only the data farther than $300 \mathrm{~m}$ is considered in the data process.

\section{B. Tuned Free-space Pass Loss Models}

In viaduct and plain scenarios, the transmitting and receiving antennas are much higher than most of the surroundings. The power of the line of sight of the radio propagation in viaduct scenario is stronger, while that of the multipath is relatively weaker. On account of this, Free-space path loss model can be used to predict the path loss of viaduct and plain scenarios. However, the difference between the measurement and Free-space path loss exists, although not big. Therefore, tuned Free-space path loss models are needed.

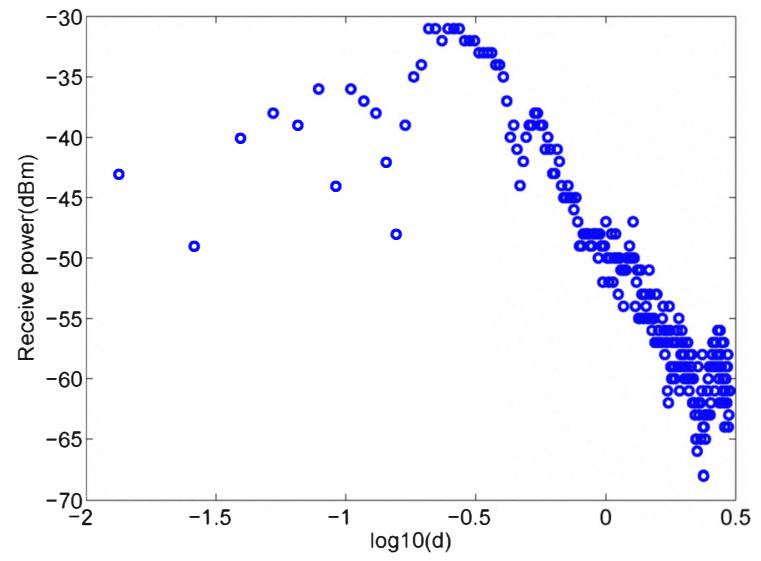

Fig. 4. Receiving power in viaduct scenario

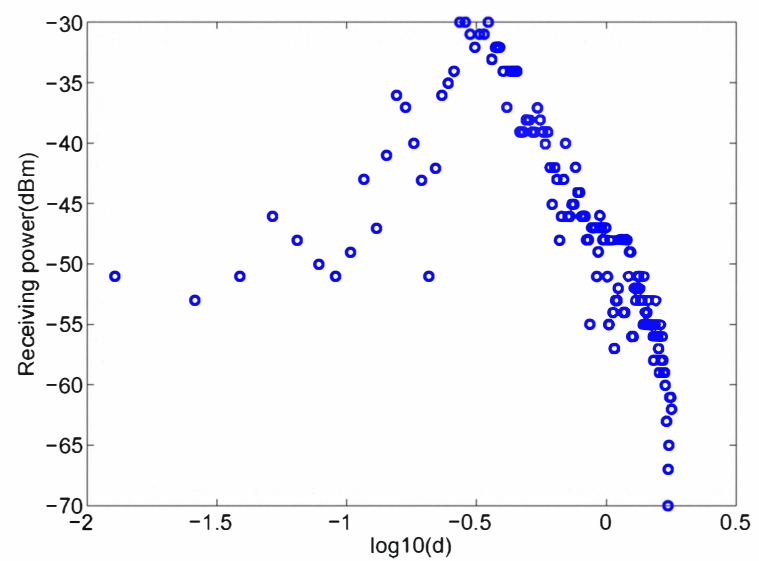

Fig. 5. Receiving power in plain scenario

Free-space path loss is calculated as:

$$
L_{0}(d B)=32.45+20 \times \log _{10}(d)+20 \times \log _{10}(f)
$$

Where, $d$ denotes distance between the transmitter and the receiver in $\mathrm{Km}$, and $f$ denotes carrier frequency in $\mathrm{MHz}$.

The receiving power is:

$$
P_{r}=P_{t}+G_{b}-l_{b}-l_{d}-L_{m}
$$

Where, $P_{r}$ is the receiving power; $P_{t}$ is the transmitting power, $P_{t}=43 \mathrm{dBm} ; G_{b}$ is the gain of the transmitting antenna, $G_{b}=17 \mathrm{dBi} ; l_{b}$ is the loss of the base station's feeder, $l_{b}=3.3 \mathrm{~dB} ; l_{d}$ is the loss of the Power-divider, $l_{d}=3.5 \mathrm{~dB}$; $L_{m}$ is the measured path loss.

Then, the path loss of the measurement data can be obtained:

$$
L_{m}=P_{t}+G_{b}-l_{b}-l_{d}-P_{r}
$$

The difference between the Free-space and measured path loss is:

$$
\Delta L=L_{m}-L_{0}
$$

On the one hand, in the measurement of each time, only the distance between the base station and the receiver changs. On the other hand, pass loss is linearly related to the distance 
in log-domain. So, the tuned equation is defined as follows:

$$
\Delta L=K_{1} \lg d+K_{2}
$$

Where, $K_{1}$ is the parameter about distance; $K_{2}$ is the constant. The two parameters are obtained by Least-square criterion.

In the modeling process, 274 groups in viaduct and 30 groups in plain of measured data are used. As the measurement data of the receiving power may sometimes invalid, the parameters $K_{1}$ and $K_{2}$ are invalid consequently. The $3 \sigma$ criterion is introduced to eliminate the invalid values of the two parameters. $\sigma$ and $\left|\Delta v_{i}\right|=\left|v_{i}-\bar{v}\right|$ denote the standard deviation and absolute value of the residuals between the data and their mean value, respectively. If the condition $\left|\Delta v_{i}\right|>$ $3 \sigma$ is satisfied, the criterion detects the invalid data. After the detection, 260 and 30 groups left in viaduct and plain scenarios. Then, the parameters' median value, mean value and standard deviation of 260 groups of $K_{1}$ and $K_{2}$ in viaduct scenario are given in Table I. And those of 30 groups of $K_{1}$ and $K_{2}$ in plain scenario are given in Table II.

TABLE I

STATISTICAL VALUES OF $K_{1}$ AND $K_{2}$ IN VIADUCT SCENARIO

\begin{tabular}{cccc}
\hline Parameter & Median $(\mathrm{dB})$ & Mean $(\mathrm{dB})$ & $\mathrm{Std}(\mathrm{dB})$ \\
\hline$K_{1}$ & 6.26 & 6.9152 & 6.019 \\
$K_{2}$ & 9.855 & 7.33 & 12.38 \\
\hline
\end{tabular}

TABLE II

STATISTICAL VALUES OF $K_{1}$ AND $K_{2}$ IN PLAIN SCENARiO

\begin{tabular}{cccc}
\hline Parameter & Median $(\mathrm{dB})$ & Mean $(\mathrm{dB})$ & Std $(\mathrm{dB})$ \\
\hline$K_{1}$ & 14.19 & 12.82 & 10.28 \\
$K_{2}$ & 12.72 & 14.9 & 5.339 \\
\hline
\end{tabular}

In this paper, the median values of the $K_{1}$ and $K_{2}$ are employed. The mean value is calculated by averaging the values of different groups. One situation that can make the things get worse is when there exits several higher values. Fortunately, the median value can avoid this problem. So, the values of $K_{1}$ and $K_{2}$ are obtained finally.

In viaduct scenario: $\mathrm{K} 1=6.26, \mathrm{~K} 2=9.855$

In plain scenario: $\mathrm{K} 1=14.19, \mathrm{~K} 2=12.72$

The tuned Free-space path loss model is:

$$
L=\Delta L+L_{0}
$$

$L=K_{1} \times \log _{10}(d)+K_{2}+32.45+20 \times \log _{10}(d)+20 \times \log _{10}(f)$

Then, the models in viaduct and plain scenarios are repressed as:

$$
\begin{gathered}
L_{v i a}=42.305+26.26 \times \log _{10}(d)+20 \times \log _{10}(f) \\
L_{p l a}=46.17+34.19 \times \log _{10}(d)+20 \times \log _{10}(f)
\end{gathered}
$$

\section{Performance Evaluation}

In this section, performance evaluation of models is done to analyze whether the tuned models can adjust the measurement data or not. To do this, open area scenario in Hata, similar to viaduct and plain scenarios of the High-speed Railway, is used for comparison. And the groups that used for model evaluation are 180 and 30 in viaduct and plain scenarios, respectively.

\section{A. Hata Model in Open Area and Introduction of the Evalu- ation Terms}

In open area, the Hata model is expressed as following:

$$
\begin{array}{r}
L_{\text {urban }}=69.55+26.26 \times \log _{10}(f)-13.82 \times \log _{10}\left(h_{b}\right) \\
-\alpha\left(h_{m}\right)+\left(44.9-6.55 \times \log _{10}\left(h_{b}\right)\right) \log _{10}(d)-K \\
\alpha\left(h_{m}\right)=\left(1.1 \times \log _{10}(f)-0.7\right) h_{m}-\left(1.56 \times \log _{10}(f)-0.8\right) \\
K=4.78 \times\left(\log _{10}(f)\right)^{2}-18.33 \times \log _{10}(f)+40.94
\end{array}
$$

Where, $f$ donates frequency; $h_{b}$ donates the height of the base station; $d$ donates the distance between the transmitter and the receiver; $\alpha\left(h_{m}\right)$ donates the correction factor of the height of the mobile station; $K$ donates the correction factor for open area.

To evaluate the performance of the models, the residuals of the path loss between the measurement and the models are studied. And the expression is shown below:

$$
\begin{array}{r}
\Delta L=L_{m}-L=\left\{\Delta L_{1}, \Delta L_{2}, \ldots \Delta L_{\left.i, \ldots \Delta L_{N}\right\}}\right. \\
i=1,2 \ldots N
\end{array}
$$

Where, $L_{m}$ is the measurement path loss; $L$ is the corresponding path loss of the models for comparison; $N$ is the total number of the sampling points in the group.

The mean value $(\mathrm{M})$ of the residuals is defined as:

$$
\overline{\Delta L}=\frac{\sum_{i=1}^{N} \Delta L_{i}}{N}
$$

The standard deviation (STD), which reflects the fluctuations of the residuals between the path loss of the measurement and the Hata model, is defined as:

$$
\sigma=\sqrt{\frac{\sum_{i=1}^{N}\left(\Delta L_{i}-\overline{\Delta L}\right)^{2}}{N-1}}
$$

Reference[12] points out that the tuned model can be used when STD is lower than $10 \mathrm{~dB}$.

And the root mean square error (RMS) is obtained as following:

$$
s=\sqrt{\frac{\sum_{i=1}^{N}\left(\Delta L_{i}\right)^{2}}{N}}
$$

\section{B. Performance Evaluation of the Two Models}

Firstly, the residuals between the tuned Free-space model and Hata model are calculated. Then, the evaluation indexes M, RMS and STD can be obtained. To observe the performance of the tuned model clearly, statistical values of M, RMS and STD in viaduct and plain scenarios are listed in Table III and Table IV. 
TABLE III

STATISTICAL VALUES OF THE RES IDUALS OF THE TUNED MODEL AND THE HATA MODEL IN VIADUCT SCENARIO

\begin{tabular}{cccc}
\hline Model & $\mathrm{M}(\mathrm{dB})$ & $\mathrm{RMS}(\mathrm{dB})$ & $\mathrm{STD}(\mathrm{dB})$ \\
\hline Tuned Free - space & 1.436 & 5.765 & 3.442 \\
Hata & 6.546 & 8.26 & 4 \\
\hline
\end{tabular}

TABLE IV

STATISTICAL VALUES OF THE RES IDUALS OF THE TUNED MODEL AND THE HATA MODEL IN PLAIN SCENARIO

\begin{tabular}{cccc}
\hline Model & $\mathrm{M}(\mathrm{dB})$ & $\mathrm{RMS}(\mathrm{dB})$ & $\mathrm{STD}(\mathrm{dB})$ \\
\hline Tuned Free - space & 2.603 & 6.379 & 4.18 \\
Hata & 13.55 & 14.09 & 3.694 \\
\hline
\end{tabular}

As the table shows, in viaduct scenario, the mean values of M, RMS and STD of the tuned model are $1.436 \mathrm{~dB}, 5.765 \mathrm{~dB}$ and $3.442 \mathrm{~dB}$, while those of Hata are $6.546 \mathrm{~dB}, 8.26 \mathrm{~dB}$ and $4 \mathrm{~dB}$. The mean error is improved by $5.1 \mathrm{~dB}$. The improvement of RMS and STD are $2.5 \mathrm{~dB}$ and $0.6 \mathrm{~dB}$, respectively. And the term STD is lower than the maximum tolerated value of $10 \mathrm{~dB}$. Therefore, the tuned model can adjust the measurement data well.

In plain scenario, the mean values of $\mathrm{M}, \mathrm{RMS}$ and STD of the tuned model are $2.603 \mathrm{~dB}, 6.379 \mathrm{~dB}$ and $4.18 \mathrm{~dB}$, while those of Hata are $13.55 \mathrm{~dB}, 14.09 \mathrm{~dB}$ and $3.694 \mathrm{~dB}$. Though the term STD of the tuned model is increased by $0.5 \mathrm{~dB}$, it shows that $\mathbf{M}$ and RMS decrease significantly after tuning process, which are $11.0 \mathrm{~dB}$ and $7.7 \mathrm{~dB}$ respectively.

For the mean error, $-10 \mathrm{~dB}$ to $10 \mathrm{~dB}$ is the interval that should be paid attention to. In viaduct scenario, the probability in this interval is 0.933 for tuned model and 0.812 for Hata model. In plain scenario, the probability in this interval is 0.933 for tuned model and 0.333 for Hata model. In plain scenario, however, STD of the tuned model turns higher. But the maximum of STD is $6 \mathrm{~dB}$, which is lower than the tolerated value of $10 \mathrm{~dB}$. And as mentioned above, it only increases by $0.5 \mathrm{~dB}$. The cost is low compared to the decrease of the mean error and the root mean square error.

Fig.6 and Fig.7 present two examples to make comparison between the tuned models and the classical Hata model in viaduct and plain scenarios. The two pictures show that the tuned models work better than Hata.

\section{CONCLUSION}

In this paper, propagation measurement data in viaduct and plain scenarios of Zhengxi High-speed Railway is analyzed and discussed. Based on these measurement data, tuned Freespace path loss models are made in viaduct and plain scenarios. Finally, the tuned models are compared to the Hata model, which is widely used in GSM network. Evaluation results show that the mean errors of the tuned models are decreased by $5.1 \mathrm{~dB}$ in viaduct scenario and $11.0 \mathrm{~dB}$ in plain scenario compared to the Hata model. And the tuned models' root mean square errors are decreased by $2.5 \mathrm{~dB}$ in viaduct scenario, $7.7 \mathrm{~dB}$ in plain scenario. Consequently, these models will be

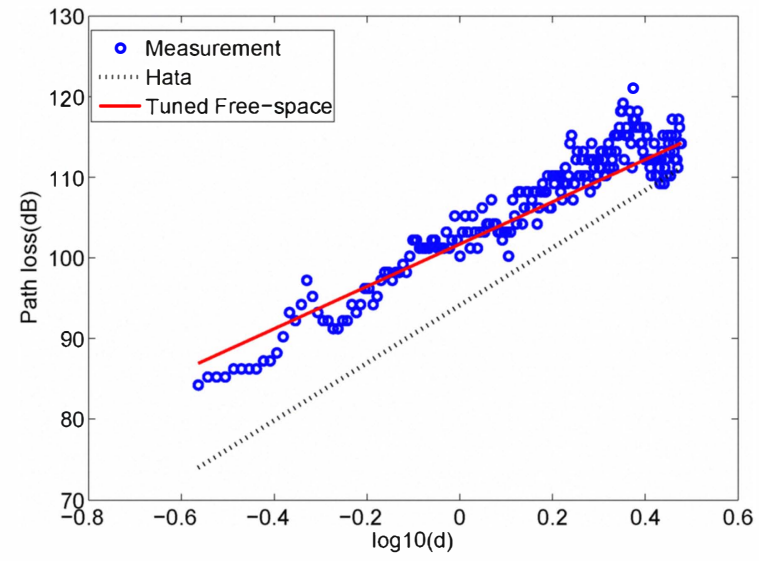

Fig. 6. Comparison of the path loss of the tuned Free-space model in viaduct, Hata model in open area and the measurement

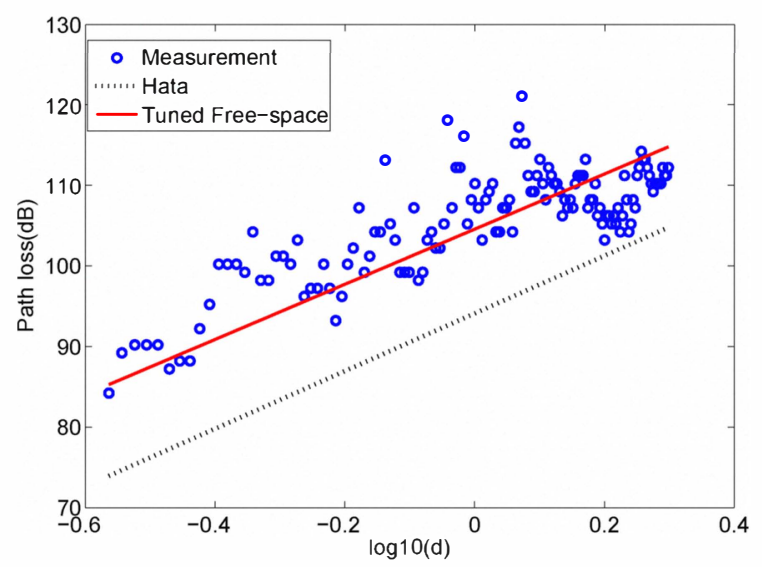

Fig. 7. Comparison of the path loss of the tuned Free-space model in plain, Hata model in open area and the measurement

useful for predicting the propagation path loss in viaduct and plain scenarios of the High-speed Railway.

\section{ACKNOWLEDGMENT}

The authors would like to thank the measurement team for their help in providing the data. Thanks also to Ruisi He and Haoxiang Zhang for data process.

\section{REFERENCES}

[1] Keith T.H., Jack W.H., David H.S., Daniel W.B., "Path-Loss Characteristics of Urban Wireless Channels," IEEE Trans. Antennas Propag., vol. 58, no.1, pp. 171-177, Jan. 2010

[2] Erceg, V., Greenstein, L.J., Tjandra, S.Y., Parkoff, S.R., Gupta, A., Kulic B., Julius, A.A., Bianchi, R., "An Empirically Based Path Loss Model forWireless Channels in Suburban Environments," IEEE J. Slelect. Areas in Commun., vol. 17, issue. 7, pp. 1205-1211, Jul. 1999.

[3] Amornthipparat A., Shirai H., Yonezawa K., Inoue T., Nakamura Y., "Estimation of High Frequency NLOS Path loss in Street-Cell Environment," Communications and Electronics, 2008. ICCE 2008. Second International Conf., pp. 336-340, Jun. 2008.

[4] P. Schneider, F. Lambrecht, A. BaierP, "Enhancement of the OkumuraHata propagation model using detailed morphological and building data," Personal, Indoor and Mobile Radio Communications, 7th IEEE International Symposium, vol. 1, pp. 34-38, Oct. 1996. 
[5] J. Turkka, M. Renfors, "Path Loss Measurements for a Non-Line-ofSight Mobile-to-Mobile Environment," ITS Telecommunications, 8th International Conf., pp. 274-278, Oct. 2008.

[6] Walden M.C., Rowsell F.J., "Urban Propagation Measurements and Statistical Path Loss Model at 3.5GHz," IEEE Antennas and Propagation Society International Symposium, vol. 1A, pp. 363-366, Jul. 2005.

[7] Hanci B.Y., Cavadar I.H., "Mobile Radio Propagation Measurements and Tuning the Path Loss Model in Urban Areas at GSM-900 Band in Istanbul-Turkey," IEEE Trans. Veh. Technol., vol. 1, pp. 139-143, Sept. 2004.

[8] Joseph W., Roelens L., Martens L., "Path Loss Model for Wireless applications at $3500 \mathrm{MHz}$," IEEE Antennas and Propagation Society International Symposium, pp. 4751-4754, Jul. 2006.
[9] Sun Kun, Wang Ping, Li Yingze, "Path Loss Models for Suburban Scenario at $2.3 \mathrm{GHz}, 2.6 \mathrm{GHz}$ and $3.5 \mathrm{GH}$," Antennas, Propagation and EM Theory, 8th International Symposium, pp. 438-441,Nov. 2008

[10] Greg Durgin, Theodore S.Rappaport, Hao Xu, "Measurements and Models for Radio Path Loss and Penetration Loss In and Around Homes and Trees at $5.85 \mathrm{GHz}$," IEEE Trans. Commun., vol. 46, Issue 11, pp.1484 - 1496, Nov. 1998.

[11] William C.Y., "Estimate of Local Average Power of a Mobile Radio signal," IEEE Trans. Veh. Technol., vol. 34, no. 1, pp. 22-27, Feb. 1985

[12] Mingjing Yang, Wenxiao Shi, "A Linear Least Square Method of Propagation Model Tuning for 3G Radio," Natural Computation, 4th International Conf., vol.5, pp. 150-154, Oct. 2008 